\title{
Maternal and infant outcomes of full-term pregnancy combined with COVID-2019 in Wuhan, China: retrospective case series
}

\author{
Yanfen Chen ${ }^{1}$. Jun Bai ${ }^{2}$ (i)
}

Received: 28 March 2020 / Accepted: 26 April 2020 / Published online: 21 July 2020

(c) Springer-Verlag GmbH Germany, part of Springer Nature 2020

\begin{abstract}
Objective To investigate the maternal and infant outcomes of full-term pregnant patients in Wuhan, China, who were infected with 2019 severe acute respiratory syndrome coronavirus 2 (SARS-CoV-2) that is responsible for coronavirus disease 2019 (COVID-2019).

Design Retrospective case series.

Setting The Central Hospitals of Wuhan, Tongji Medical College, Huazhong University of Science and Technology in Wuhan, China.

Participants Twenty one full-term pregnant patients who were admitted to the Central Hospital of Wuhan, Tongji Medical College, Huazhong University of Science and Technology, confirmed SARS-CoV-2 infection and COVID-2019 with laboratorial and clinical methods, were reviewed by our medical team, and the data were collected from January 20, 2020 to February 29, 2020.

Main clinical data collection Clinical data had been collecting using a standard case report form, such as epidemiological history, clinical manifestations, auxiliary examination of major laboratory and clinic, etc. All the information was collected and confirmed by our medical team.

Results Twenty one full-term pregnant patients were reviewed (median age 29 years), and no patients were admitted to intensive care unit (ICU), and died during the treating progress. According to our review, all the cases were infected by human to human transmission, and the most common symptoms at onset of illness were cough in 17 (80.95\%), fatigue in 10 (47.62\%), fever in 7 (33.33\%), expectoration in $1(4.76 \%)$, and only one patient (4.76\%) developed shortness of breath on admission. The median time from exposure to onset of illness was 10 days (interquartile range 7-2 days), and from onset of symptoms to first hospital admission was 1 day (interquartile range 1-2 days).

Conclusions As of February 29, 2020, all the patients who were full-term pregnancy combined with COVID-2019 were cured and delivered successfully, and all the newborns were not infected with SARS-CoV-2, and there were no evidence of mother-to-child transmission.
\end{abstract}

Keywords Full-term pregnant patients $\cdot$ SARS-CoV-2 $\cdot$ COVID-2019

Jun Bai

baijunjinanu@163.com

1 Department of Obstetrics, The Center Hospital of Wuhan, Tongji Medical College, Huazhong University of Science and Technology, Wuhan 430000, People's Republic of China

2 Department of Obstetrics and Gynecology, The First People's Hospital of Datong, 336 Hengan Avenue, Datong 037000, People's Republic of China

\section{Introduction}

In December 2019, a group of patients infected by SARSCoV-2 that is responsible for COVID-2019 in Wuhan, China. SARS-CoV-2 is similar to the coronavirus which was responsible for severe acute respiratory syndrome (SARS), and possess strong affinity to human respiratory receptors [1]. As of February 29, 2020, 48,577 COVID-2019 patients were confirmed with clinical and laboratorial methods in Wuhan, and the mortality was $4.47 \%$, and these figures are updated daily and expected to increase further [2]. COVID2019 have been drawing global attention because of the 
rapidly increasing numbers of new cases, and the transmission through human to human via droplets, contacts and aerosols.

However little is known about the maternal and infant outcomes of full-term pregnant patients combined with COVID-2019, including clinical characteristics, laboratorial findings and mother-to-child vertical transmission. Despite the increasing number of confirmed cases, the clinical investigation of full-term pregnant patients was insufficient. A previous case reported the clinical characteristics of three full-term pregnant patients infected by SARS-CoV-2 in Wuhan, contributing to an understanding of the epidemiological, clinical, laboratorial and radiological characteristics, treatment and clinical outcomes of those patients [3]. Consequently, over time more full-term pregnant patients who were infected by SARS-CoV-2 were expected to emerge across China and perhaps the world.

We are very eager to know the epidemiology, clinical manifestations, laboratorial and clinical examinations, and the presence or absence of mother-to-child transmission in full-term pregnant patients combined with COVID-2019. In this retrospective case series, we described the clinical characteristics and laboratorial findings of 21 full-term pregnant patients combined with COVID-2019 in the Central Hospitals of Wuhan, Tongji Medical College, Huazhong University of Science and Technology in Wuhan, China, and provided an insight into the prevention and treatment of full-term pregnant patients combined with COVID-19 across China and elsewhere.

\section{Methods}

\section{Data sources}

We conducted the retrospective research focusing on the maternal and infant outcomes of full-term pregnant patients combined with COVID-2019 confirmed by laboratorial and clinical methods from January 20, 2020 to February 29, 2020 in the Central Hospital of Wuhan, Tongji Medical College, Huazhong University of Science and Technology in Wuhan, China [4]. The normally diagnosing measures included full-term pregnant patients presenting with a fever, fatigue or any respiratory symptoms, including cough, expectoration, shortness of breath, transverse lung computed tomograms (CT) indicating ground glass opacity and consolidation of lower lobe of right lung near the pleura, realtime polymerase chain reaction (RT-PCR) demonstrating the nucleic acid of SARS-CoV-2 of nasopharyngeal swabs [5].

Information including illness onset, visiting clinical facilities, hospital admissions and the epidemiological data were collected by brief interviews with each patient [6]. The exposure histories of each patient during the 2 weeks before illness onset were collected, including the dates and times of close contact (gathering, living, or working together) with individuals confirmed or suspected SARS-CoV-2 infection [7].

The data of the full-term pregnant patients combined with COVID-2019 were collected and reviewed by our medical team. Because of the urgent need to collect data on this emerging pathogen, the requirement for informed consent was waived. A standard case report form to collect clinical data was adopted by our medical team.

\section{Laboratory confirmation and treatment}

The nucleic acid of SARS-CoV-2 of sputum and throat swab specimens collected from all patients at admission were detected by RT-PCR within $3 \mathrm{~h}$, and the detection was repeated twice every $24 \mathrm{~h} \mathrm{[8].} \mathrm{Lung} \mathrm{CT} \mathrm{was} \mathrm{re-conducted}$ every 2 days among all the pregnant patients at admission [9]. Laboratory tests were performed at admission including a complete blood count, serum biochemistry, and identification of other respiratory pathogens such as influenza A/B virus, respiratory syncytial virus, parainfluenza virus, and adenovirus $[8,9]$.

All patients had been received antiviral treatment with Ribavirin (500 mg twice daily), or Arbidol (200 mg twice daily) for 10 days [10]. Dexamethasone (10 mg/day) had been administrated to patients whose fetal lungs were evaluated as premature for 2 days [3]. Heparin Sodium (2500 IU/ day) had been used to prevent thrombosis for 5 days [10]. Ceftezole sodium ( $2 \mathrm{~g}$ twice daily) and Ceftriaxone sodium ( $2 \mathrm{~g}$ twice daily) had been implemented via intravenous drip to prevent bacterial infections after cesarean section for 5 days. Oxytocin ( $20 \mathrm{U}$ one daily) had been used to promote uterine instauration after cesarean section [11]. Diamine glycyrrhizinate enteric capsules ( $150 \mathrm{mg}$ triple daily) had been taken orally for 7 days when serum biochemistry indicated liver function impaired [12]. Once the fetus was evaluated as mature, the pregnancy will be terminated by cesarean section [12].

\section{Statistical analysis}

The continuous variables as medians with interquartile ranges were summarized. The percentages of patients in each category for categorical variables were calculated. All analyses were done with SPSS software, version 20.0.

\section{Patient and public involvement}

This was a retrospective case series study and no patients were involved in the study design, setting the research questions, or the outcome measures directly. No patients were asked to advise interpretation or writing up of results. 


\section{Results}

\section{Epidemiological characteristics}

As of February 29, 2020, the clinical data of 21 full-term pregnant patients combined with COVID-2019 confirmed with laboratorial and clinical methods in the Central Hospital of Wuhan, Tongji Medical College, Huazhong University of Science and Technology in Wuhan, China. $10(47.62 \%)$ of the patients were aged $22-28$ years, ten $(47.62 \%)$ were aged $29-34$ years, one $(4.76 \%)$ were aged 35 years and older. The median age was 29 years (interquartile range 27-31 years; Table 1). As of January 20, 2020, all 21 patients had been exposed to individuals confirmed SARS-CoV-2 infection. All the 21 patients resided in Wuhan before illness onset, and 17 (80.95\%) patients could provide the exact date of close contact with someone confirmed or suspected SARS-CoV-2 infection.

\section{Clinical features}

11 patients (52.38\%) had no any underlying diseases, three (14.29\%) combined with anemia, three (14.29\%) gestational diabetes, two (9.52\%) hypothyroidism, and two (9.52\%) hepatitis B virus carriers (Table 1$)$. Two patients $(9.52 \%)$ had premature rupture of membranes and got natural delivery successfully (Table 1). 17 patients who could provide the exact date of close contact with someone confirmed or suspected SARS CoV-2 infection, the median incubation period from exposure to symptoms was 10 days (interquartile range 7-12 days; Table 1). The median time from onset of symptoms to first hospital admission was 1 day (interquartile range 1-2 days; Table 1). The most common symptoms at illness onset were cough in $17(80.95 \%)$, fatigue in 10 (47.62\%), fever in 7 (33.33\%), expectoration in $1(4.76 \%)$, and only (4.76\%) patient developed shortness of breath (Table 1).

On admission, the blood counts of one $(4.76 \%)$ patient showed leucopenia (white blood cell count $<6 \times 109 / \mathrm{L}$ )

Table 1 Personal and clinical characteristics of 21 full-term pregnant patients combined with COVID-2019 in the Center Hospital of Wuhan, Tongji Medical College, Huazhong University of Science and Technology in Wuhan, China

\begin{tabular}{|c|c|c|c|}
\hline \multirow[t]{2}{*}{ Characteristics } & \multirow[t]{2}{*}{ All patients $(n=21)$} & \multicolumn{2}{|c|}{ Time since symptom onset } \\
\hline & & $>10$ days $(n=17)$ & $\leq 10$ days $(n=4)$ \\
\hline Median (interquartile) age (years) & $29(27-31)$ & $31(29.50-33.50)$ & $25(23.50-26.50)$ \\
\hline \multicolumn{4}{|l|}{ Age groups (years) } \\
\hline $22-28$ & $10(47.62)$ & $6(35.29)$ & $4(100.00)$ \\
\hline $29-34$ & $10(47.62)$ & $10(58.82)$ & $0(0)$ \\
\hline$\geq 35$ & $1(4.76)$ & $1(5.88)$ & $0(0)$ \\
\hline \multicolumn{4}{|l|}{ Complications } \\
\hline Anemia & $3(14.29)$ & $2(11.77)$ & $1(25.00)$ \\
\hline Hypothyroidism & $2(9.52)$ & $2(11.77)$ & $0(0)$ \\
\hline Gestational diabetes & $3(14.29)$ & $3(17.65)$ & $0(0)$ \\
\hline Hepatitis B virus carriers & $2(9.52)$ & $2(11.77)$ & $0(0)$ \\
\hline Premature rupture of membranes & $6(28.57)$ & $5(29.41)$ & $1(25.00)$ \\
\hline Familial cluster & $2(9.52)$ & $2(11.77)$ & $0(0)$ \\
\hline Fever & $7(33.33)$ & $6(35.29)$ & $1(25.00)$ \\
\hline \multicolumn{4}{|l|}{ Highest temperature $\left({ }^{\circ} \mathrm{C}\right)$} \\
\hline$\leq 37.3$ & $1(4.76)$ & $1(5.88)$ & $0(0)$ \\
\hline $37.3-38.0$ & $5(23.81)$ & $4(23.53)$ & $1(25.00)$ \\
\hline $38.1-39.0$ & $1(4.76)$ & $1(5.88)$ & $0(0)$ \\
\hline Respiratory rate $>24$ breaths per min & $1(4.76)$ & $1(5.88)$ & $0(0)$ \\
\hline Oxygenation index & $2(9.52)$ & $2(11.77)$ & $0(0)$ \\
\hline Cough & $17(80.95)$ & $16(94.12)$ & $1(25.00)$ \\
\hline Fatigue & $10(47.62)$ & $9(52.94)$ & $1(25.00)$ \\
\hline Expectoration & $1(4.76)$ & $1(5.88)$ & $0(0)$ \\
\hline Median (interquartile) incubation period (days) & $10(7-12)$ & $9(7-12)$ & $11(9.25-12.25)$ \\
\hline $\begin{array}{l}\text { Median (interquartile) time from illness onset to first hos- } \\
\text { pital admission (days) }\end{array}$ & $1(1-2)$ & $1(1-2)$ & $1.5(1-2)$ \\
\hline
\end{tabular}

Percentages do not total $100 \%$ owing to missing data. Values are numbers (percentages) unless stated otherwise 
and four (19.05\%) showed lymphopenia (lymphocyte count $<1.0 \times 109 / \mathrm{L}$ ), Aspartate aminotransferase of one $(4.76 \%)$ patient was upregulated $(\geq 40 \mathrm{U} / \mathrm{L})$, creatine kinase of two (9.52\%) patients was raised ( $\geq 185 \mathrm{U} / \mathrm{L})$, Lactate dehydrogenase of two (9.52\%) patients was upregulated (> 245 U/L), Procalcitonin of nine (42.86\%) patients went up ( $\geq 0.1 \mathrm{ng} / \mathrm{mL}$; Table 2). The levels of creatine, D-dimer, alanine aminotransferase, platelet count and haemoglobin

Table 2 Laboratory and lung radiography findings in full-term pregnant patients combined with COVID-2019 in the Center Hospital of Wuhan, Tongji Medical College, Huazhong University of Science and Technology in Wuhan, China

\begin{tabular}{|c|c|c|c|c|}
\hline \multirow[t]{2}{*}{ Variables } & \multirow[t]{2}{*}{ All patients $(n=21)$} & \multicolumn{2}{|l|}{ Time since symptom onset } & \multirow[t]{2}{*}{ Normal range } \\
\hline & & $>10$ days $(n=17)$ & $\leq 10$ days $(n=4)$ & \\
\hline White blood cell count $\left(\times 10^{9} / \mathrm{L}\right)$ & $7.58(6.61-11.22)$ & $7.58(6.61-11.22)$ & $8.13(6.74-10.61)$ & $6.00-20.00$ \\
\hline \multicolumn{5}{|l|}{ White blood cell count $\left(\times 10^{9} / \mathrm{L}\right)($ no $(\%))$} \\
\hline$\leq 6$ & $1(4.76)$ & $1(5.88)$ & $0(0)$ & \\
\hline $6-20$ & $18(85.71)$ & $14(82.35)$ & $4(100.00)$ & \\
\hline$>20$ & $2(9.52)$ & $2(11.77)$ & $0(0)$ & \\
\hline Neutrophil count $\left(\times 10^{9} / \mathrm{L}\right)$ & $5.87(4.68-9.26)$ & $5.87(4.68-9.26)$ & $6.13(4.57-8.39)$ & $1.80-6.30$ \\
\hline Lymphocyte count $\left(\times 10^{9} / \mathrm{L}\right)$ & $1.41(1.17-1.86)$ & $1.39(1.12-1.67)$ & $1.76(1.36-2.14)$ & $1.10-3.20$ \\
\hline \multicolumn{5}{|l|}{ Lymphocyte count $\left(\times 10^{9} / \mathrm{L}\right)($ no $(\%))$} \\
\hline$\leq 1.0$ & $4(19.05)$ & $4(23.53)$ & $0(0)$ & \\
\hline$>1.0$ & $17(80.95)$ & $13(76.47)$ & $4(100.00)$ & \\
\hline Haemoglobin $(\mathrm{g} / \mathrm{L})$ & $120.00(112.00-129.00)$ & $119.00(112.00-129.00)$ & $128.50(112.00-132.25)$ & $100.00-130.00$ \\
\hline Platelet count $\left(\times 10^{9} / \mathrm{L}\right)$ : & $175.00(166.00-245.00)$ & $175.00(161.00-222.00)$ & $219.50(174.25-275.50)$ & $125.00-350.00$ \\
\hline \multicolumn{5}{|l|}{ Platelet count $\left(\times 10^{9} / \mathrm{L}\right)($ no $(\%))$} \\
\hline$\leq 100$ & $0(0)$ & $0(0)$ & $0(0)$ & \\
\hline$>100$ & $21(100.00)$ & $17(100.00)$ & $4(100.00)$ & \\
\hline D-dimer (mg/L) & $2.99(1.74-4.60)$ & $2.99(1.74-4.46)$ & $4.54(2.08-6.59)$ & $<0.50$ \\
\hline Alanine aminotransferase (U/L) & $9.5(7.10-10.90)$ & $9.80(7.10-10.90)$ & $8.15(7.00-10.33)$ & $7.00-40.00$ \\
\hline Aspartate aminotransferase (U/L) & $15.50(13.00-20.10)$ & $15.50(13.40-20.10)$ & $13.80(11.75-17.45)$ & $13.00-35.00$ \\
\hline \multicolumn{5}{|l|}{ Aspartate aminotransferase (U/L) (no (\%)) } \\
\hline$\leq 40$ & $20(95.24)$ & $16(94.12)$ & $4(100.00)$ & \\
\hline$>40$ & $1(4.76)$ & $1(5.88)$ & $0(0)$ & \\
\hline Potassium (mmol/L) & $4.05(3.89-4.24)$ & $4.05(3.89-4.11)$ & $4.17(3.87-4.29)$ & $3.50-5.30$ \\
\hline Sodium (mmol/L) & $137.7(136.50-139.50)$ & $137.90(136.70-140.10)$ & $135.95(134.43-137.45)$ & $137.00-147.00$ \\
\hline Creatine $(\mu \mathrm{mol} / \mathrm{L})$ & $43.40(35.60-46.20)$ & $43.50(36.70-46.20)$ & $38.95(33.48-50.55)$ & $41.00-73.00$ \\
\hline \multicolumn{5}{|l|}{ Creatine $(\mu \mathrm{mol} / \mathrm{L})($ no $(\%))$} \\
\hline$\leq 133$ & $21(100.00)$ & $17(100.00)$ & $4(100.00)$ & \\
\hline$>133$ & $0(0)$ & $0(0)$ & $0(0)$ & \\
\hline Creatine kinase (U/L) & $51.00(36.00-71.00)$ & $52.00(35.00-78.00)$ & $45.50(41.75-53.75)$ & $40.00-200.00$ \\
\hline \multicolumn{5}{|l|}{ Creatine kinase (U/L) (no (\%)) } \\
\hline$\leq 185$ & $19(90.48)$ & $15(88.24)$ & $4(100.00)$ & \\
\hline$>185$ & $2(9.52)$ & $2(11.77)$ & $0(0)$ & \\
\hline Lactate dehydrogenase (U/L) & $152.00(132.00-175.00)$ & $152.00(132.00-175.00)$ & $152.00(142.50-164.00)$ & $120.00-250.00$ \\
\hline \multicolumn{5}{|l|}{ Lactate dehydrogenase (U/L) (no (\%)) } \\
\hline$\leq 245$ & $19(90.48)$ & $15(88.24)$ & $4(100.00)$ & \\
\hline$>245$ & $2(9.52)$ & $2(11.77)$ & $0(0)$ & \\
\hline Procalcitonin (ng/mL) & $0.06(0.04-0.14)$ & $0.06(0.05-0.14)$ & $0.08(0.04-0.14)$ & $0.00-0.05$ \\
\hline \multicolumn{5}{|l|}{ Procalcitonin (ng/mL) (no (\%)) } \\
\hline$\leq 0.1$ & $12(57.14)$ & $9(52.94)$ & $3(75.00)$ & \\
\hline$>0.1$ & $9(42.86)$ & $8(47.06)$ & $1(25.00)$ & \\
\hline Bilateral involvement on chest radiographs & $21(100.00)$ & $17(100.00)$ & $4(100.00)$ & \\
\hline Pneumonia & $21(100.00)$ & $17(100.00)$ & $4(100.00)$ & \\
\hline
\end{tabular}

Percentages do not total $100 \%$ owing to missing data. (Values are medians (interquartile ranges) unless stated otherwise 
were within normal range (Table 2). All 21 (100\%) patients showed were bilateral or multiple lobular or sub-segmental areas of consolidation or bilateral ground glass opacity on lung CT (Table 2). All patients were received antiviral treatment, and 19 (90.48\%) were given empirical antibiotic treatment, and $4(19.05 \%)$ were treated systematic dexamethasone treatment for promoting fetal lung maturation, and $2(9.52 \%)$ were acquired protecting liver treatment, and $1(4.76 \%)$ was achieved anticoagulation treatment. At this point, all pregnant patients had been discharged and no patients had died. Fitness for discharge was based on abatement of fever for at least 3 days, with improved evidence on lung radiography and viral clearance in samples from the lower respiratory tract (Table 3).

\section{Maternal and infant outcomes}

All the full-term pregnant patients combined with COVID2019 were received a successful delivery, 16 patients terminated pregnancy by cesarean section, and five got natural deliveries. The nucleic acid of SARS-CoV-2 of nasopharyngeal swab of newborns was detected using RT-PCR, and all the results were negative, lung CT was performed according to the clinical guidelines of COVID-2019, and there were no any infected signs in all the newborns' lungs. All in all, there were no any evidence on mother-to-child vertical transmission. As a safety precaution, all newborns and their mothers had been isolated for 14 days, and all newborns were not breastfed during the 14 days of isolation period with their mother, and the mothers and newborns were not permitted to share rooms together until the mothers had been rediagnosed no SARS-CoV-2 infection after they had been cured and subsequently isolated for 14 days.

\section{Discussion}

As of February 29, 2020, the cumulatively confirmed patients of COVID-2019 were 48,557, the presently confirmed patients were 28,836 , the patients who had died were 2169 , the cured patients were 17,552 , and the mortality rate was $4.47 \%$, which were reported in Wuhan, China [2]. Now the Chinese biologists have had a fully understanding of SARS-CoV-2, including biological and epidemiological characteristics, even the vaccine of SARS-CoV-2 has been developing. Chinese clinical therapists have already had maturely diagnosing guidelines and treating scheme on COVID-2019 [13]. But the effect of COVID-2019 on full-term pregnant patients, the treating scheme of full-term pregnancy combined with COVID-2019 and the mother-tochild transmission are still not reported [14].

In our study, Ribavirin and Arubidol were regarded to have the potential to treat COVID-2019 and administrated to the patients as an empirical medication, which were supposed to be a beneficial part of the treatment for full-term pregnant patients combined with COVID-2019 [15]. Ceftezole sodium and Ceftriaxone were used to prevent bacterial infections when the pregnant patients were received a cesarean section [16]. Dexamethasone promoting fetal lung maturation was also applicable to full-term patients combined with COVID-2019 [3]. Heparin Sodium was used to prevent thrombosis and Diamine glycyrrhizinate was administrated to protect liver function $[3,15,16]$. All the patients who had
Table 3 Treatments and outcomes in full-term pregnant patients combined with COVID2019 in the Center Hospital of Wuhan, Tongji Medical College, Huazhong University of Science and Technology in Wuhan, China

\begin{tabular}{llll}
\hline Treatments and outcomes & All patients $(n=21)$ & \multicolumn{2}{l}{ Time since symptom onset } \\
\cline { 3 - 4 } & & $>10$ days $(n=17)$ & $\leq 10$ days $(n=4)$ \\
\hline Admission to intensive care unit & $0(0)$ & $0(0)$ & $0(0)$ \\
Acute respiratory distress syndrome & $0(0)$ & $0(0)$ & $0(0)$ \\
Treatment & & & \\
Antiviral treatment & $21(100.00)$ & $17(100.00)$ & $4(100.00)$ \\
Ribavirin & $13(61.91)$ & $12(70.59)$ & $1(25.00)$ \\
Arbidol & $8(38.10)$ & $5(29.41)$ & $3(75.00)$ \\
Ribavirin +arbidol & $0(0)$ & $0(0)$ & $0(0)$ \\
Antibiotics & $19(90.48)$ & $16(94.12)$ & $3(75.00)$ \\
Dexamethasone & $4(19.05)$ & $3(17.65)$ & $1(25.00)$ \\
Anti-liver damage & $2(9.52)$ & $2(11.77)$ & $0(0)$ \\
Antithrombotic & $1(4.76)$ & $1(5.88)$ & $0(0)$ \\
Prognosis & & & $4(100.00)$ \\
Hospital admission & $21(100.00)$ & $17(100.00)$ & $4(100.00)$ \\
Discharge & $21(100.00)$ & $17(100.00)$ & $0(0)$ \\
Death & $0(0)$ & $0(0)$ & \\
\hline
\end{tabular}

Percentages do not total $100 \%$ owing to missing data. Values are numbers (percentages) of patients 
underlying diseases, such as anemia, gestational diabetes and so on, were received normal treatment according to the guidelines for clinical treatment in obstetrics. Whether the use of antivirals, antibiotics, or antithrombotic affected the prognosis of patients remains unknown, but all the medical intervention received good clinical results, all COVID-2019 were cured, all patients got a satisfied delivery and there was no any evidence of mother-to-child transmission.

By the observation and treatment of the full-term pregnant patients combined with COVID-2019, we found that the disease courses of the full-term pregnant patients combined with COVID-2019 were longer than the non-pregnant patient combined with COVID-2019 in Wuhan, but the clinical symptoms of the full-term pregnant patients combined with COVID-2019 were less severe than the non-pregnant patients combined with COVID-2019 in Wuhan. All the patients had been cured and got a successfull delivery. Why this clinical phenomenon happened, maybe zygote implant in endometrial tissue, and grow into embryo and fetus, which is a kind of semi-allogenic graft, a great numbers of immune response occur, and create a lot of cytokines that protect embryo and fetus growth, adequate cytokine accumulate during third trimester, which may possess anti-SARS-CoV-2 infecting ability. Placental barrier may block SARS-CoV-2 to enter embryo and fetus through placenta, protect embryo and fetus from SARS-CoV-2 infection. Maybe SARS-CoV-2 has no infecting ability to human fetus during third trimester, just as severe acute respiratory syndrome (SARS), and other human coronavirus [17]. Younger full-time pregnant patients combined with COVID-2019 had relatively longer incubation period and fast prognosis compared with older patients, the full-term pregnant patients combined with COVID-2019 who possessed underlying diseases had more severe clinical symptoms and longer treated time compared with full-term pregnant patients, which demonstrated the ability to resist COVID-2019 decreases with age in adult pregnant women, the ability to repel COVID-2019 declines with underlying diseases.

But this study had several limitations. Firstly, only 21 patients were included in our research and clinical treatment, while a large number of full-term pregnant patients combined with COVID-2019 were continually being admitted to other hospital as those data were not being collected. Secondly, the patients' symptoms who were mild or moderate in our hospital, and only one patient had dyspnea, and one patient developed acute respiratory distress syndrome and was admitted in an intensive care unit. The results of laboratorial test and lung $\mathrm{CT}$ also showed that the patients experienced mild and moderate illness. Whether there were mother-to-child vertical transmission among full-term pregnant patients combined with COVID-2019 who had the severe symptoms or not, which is still unknown. Moreover, the time since illness onset in some of our patients might be shorter than the observation period of 10 days, which could result in biases of clinical observation characteristics.

\section{Conclusion}

The full-term pregnant patients combined with COVID2019 with mild and moderate symptoms in Wuhan, China in our study could be cured, and got successful delivery, and there were no evidence of mother-to-child transmission (PMTCT) currently.

Acknowledgements The authors would like to thank Professor Yanping Gao (The First People's Hospital of Datong, Datong, Shanxi, China) for her clinical direction of study design.

Author contribution YC: Study Design, Data Collection. JB: Study Design, Statistical Analysis, Manuscript Preparation.

Funding This research has no funding resource.

\section{Compliance with ethical standards}

Conflict of interest The authors declare no competing or financial interests.

\section{References}

1. Xu XW, Wu XX, Jiang XG et al (2020) Clinical findings in a group of patients infected with the 2019 novel coronavirus (SARS-Cov-2) outside of Wuhan, China: retrospective case series. BMJ 368:m606. https://doi.org/10.1136/bmj.m792

2. National health commission (2020) Update on new coronavirus pneumonia as at 24:00 hours on 29 February. https://ncov.dxy.cn/ ncovh5/view/pneumonia

3. Chen S, Huang B, Luo DJ et al (2020) Pregnant women with new coronavirus infection: a clinical characteristics and placental pathological analysis of three cases. Zhonghua Bing Li Xue Za Zhi 49:E005. https://doi.org/10.3760/cma.j.cn112151-20200 225-00138

4. Bajema KL, Oster AM, McGovern OL et al (2020) (2020) Persons evaluated for 2019 novel coronavirus-United States. MMWR Morb Mortal Wkly Rep 69(6):166-170. https://doi.org/10.15585/ mmwr.mm6906e1

5. Ryu S, Chun BC (2020) An interim review of the epidemiological characteristics of 2019 novel coronavirus. Epidemiol Health 42:e2020006. https://doi.org/10.4178/epih.e2020006

6. Habibzadeh P, Stoneman EK (2020) The novel coronavirus: a bird's eye view. Int J Occup Environ Med 11(2):65-71. https:// doi.org/10.15171/ijoem.2020.1921

7. Tong ZD, Tang A, Li KF et al (2020) Potential presymptomatic transmission of SARS-CoV-2, Zhejiang Province, China, 2020. Emerg Infect Dis. https://doi.org/10.3201/eid2605.200198

8. Chen TM, Rui J, Wang QP et al (2020) A mathematical model for simulating the phase-based transmissibility of a novel coronavirus. Infect Dis Poverty 9(1):24. https://doi.org/10.1186/s4024 9-020-00640-3

9. Hellewell J, Abbott S, Gimma A et al (2020) Feasibility of controlling COVID-19 outbreaks by isolation of cases and 
contacts. Lancet Glob Health. https://doi.org/10.1016/S2214 $-109 X(20) 30074-7$

10. Liu Y, Eggo RM, Kucharski AJ (2020) Secondary attack rate and superspreading events for SARS-CoV-2. Lancet 395(10227):e47. https://doi.org/10.1016/S0140-6736(20)30462-1

11. Rasmussen SA, Smulian JC, Lednicky JA et al (2020) Coronavirus disease 2019 (COVID-19) and pregnancy: what obstetricians need to know. Am J Obstet Gynecol. https://doi.org/10.1016/j. ajog.2020.02.017

12. Fu X, Ying Q, Zeng T et al (2020) Simulating and forecasting the cumulative confirmed cases of SARS-CoV-2 in China by boltzmann function-based regression analyses. J Infect. https://doi. org/10.1016/j.jinf.2020.02.019

13. Huang Y, Tu M, Wang S et al (2020) Clinical characteristics of laboratory confirmed positive cases of SARS-CoV-2 infection in Wuhan, China: a retrospective single center analysis. Travel Med Infect Dis. https://doi.org/10.1016/j.tmaid.2020.101606

14. Xu YH, Dong JH, An WM et al (2020) Clinical and computed tomographic imaging features of novel coronavirus pneumonia caused by SARS-CoV-2. J Infect. https://doi.org/10.1016/j. jinf.2020.02.017
15. Lana RM, Coelho FC, Gomes MFDC et al (2020) The novel coronavirus (SARS-CoV-2) emergency and the role of timely and effective national health surveillance. Cad Saude Publica 36(3):e00019620. https://doi.org/10.1590/0102-311x00019620

16. Ahmed SF, Quadeer AA, McKay MR (2020) Preliminary identification of potential vaccine targets for the COVID-19 coronavirus (SARS-CoV-2) based on SARS-CoV immunological studies. Viruses 12(3):E254. https://doi.org/10.3390/v12030254

17. Xu J, Zhao S, Teng T et al (2020) Systematic comparison of two animal-to-human transmitted human coronaviruses: SARS-CoV-2 and SARS-CoV. Viruses 12(2):E244. https://doi.org/10.3390/ v12020244

Publisher's Note Springer Nature remains neutral with regard to jurisdictional claims in published maps and institutional affiliations. 